\title{
Bilinear Equation of the Nonlinear Partial Differential Equation and Its Application
}

\author{
Xiao-Feng Yang $\mathbb{D}^{1}$ and Yi Wei $\mathbb{D}^{2}$ \\ ${ }^{1}$ College of Science, Northwest A\&F University, Yangling, 712100 Shaanxi, China \\ ${ }^{2}$ School of Medical Information Engineering, Jining Medical University, Rizhao 276826, China \\ Correspondence should be addressed to Xiao-Feng Yang; yangxiaofeng@nwafu.edu.cn
}

Received 4 March 2020; Accepted 6 April 2020; Published 28 April 2020

Academic Editor: Liguang Wang

Copyright (C) 2020 Xiao-Feng Yang and Yi Wei. This is an open access article distributed under the Creative Commons Attribution License, which permits unrestricted use, distribution, and reproduction in any medium, provided the original work is properly cited.

\begin{abstract}
The homogeneous balance of undetermined coefficient method is firstly proposed to derive a more general bilinear equation of the nonlinear partial differential equation (NLPDE). By applying perturbation method, subsidiary ordinary differential equation (subODE) method, and compatible condition to bilinear equation, more exact solutions of NLPDE are obtained. The KdV equation, Burgers equation, Boussinesq equation, and Sawada-Kotera equation are chosen to illustrate the validity of our method. We find that the underlying relation among the $\left(G^{\prime} / G\right)$-expansion method, Hirota's method, and HB method is a bilinear equation. The proposed method is also a standard and computable method, which can be generalized to deal with other types of NLPDE.
\end{abstract}

\section{Introduction}

The nonlinear partial differential equation (NLPDE) is known to describe a wide variety of phenomena not only in physics but also in biology, chemistry, and several other fields [1-3]. The investigation of the exact solutions for NLPDE plays an important role on the study of nonlinear physical phenomena [4-9]. In recent years, many powerful methods are used to obtain the exact solutions of NLPDE, for example, the inverse scattering method [7], Bäcklund and Darboux transformation method [8], homotopy perturbation method [9], first integral method [10-12], variational iteration method [13, 14], sub-ODE method [15-17], Jacobi elliptic function method [18], tanh-sech method [19], $\left(G^{\prime} / G\right)$ expansion method [20, 21], Hirota's method [22-24], and homogeneous balance (HB) method [25-27].

As the direct methods, the $\left(G^{\prime} / G\right)$-expansion method, Hirota's method, and HB method are very effective for constructing the exact solutions of NLPDE. Exact traveling wave solutions, $\mathrm{N}$-soliton solutions, and solitary wave solutions of some NLPDE are obtained by using the above three methods [20-30]. Fan improved the HB method to investigate the BT, Lax pairs, symmetries, and exact solutions for some NLPDE [31, 32]. He also showed that there are many links among the HB method, Weiss-Tabor-Carnevale method, and Clarkson-Kruskal method.

However, there is no unified direct method which can be used to deal with all types of NLPDE. And also, no literature is available to illustrate the underlying relations among the three direct methods.

In the present paper, by improving some key steps in the HB method [26], we propose a new method, HB of undetermined coefficient method, which can be used to derive the bilinear equation of NLPDE. Based on the bilinear equation, by applying the perturbation method, sub-ODE method, and compatible condition, more exact solutions of NLPDE are obtained. We illustrate the real meaning of balance numbers. We show the underlying relations among the $\left(G^{\prime} / G\right)$-expansion method, Hirota's method, and HB method.

This paper is organized as follows: the HB of undetermined coefficient method is described in Section 2. In Sections 3 and 4, the KdV equation and Burgers equation are chosen as examples to illustrate the method, respectively. In Section 5, the bilinear equations of Boussinesq equation and Sawada-Kotera equation are derived, respectively. Some brief conclusions are given in Section 6. 


\section{Description of the HB of Undetermined Coefficient Method}

Let us consider a general NLPDE, say, in two variables

$$
P\left(u, u_{t}, u_{x}, u_{x x}, u_{x t}, \cdots\right)=0 \text {, }
$$

where $P$ is a polynomial function of its arguments and the subscripts denote the partial derivatives. The $\mathrm{HB}$ of undetermined coefficient method consists of three steps.

Step 1. Suppose that the solution of Equation (1) is of the form

$$
u=a_{m n}(\ln w)_{m, n}+\sum_{\substack{i, j=0 \\ i+j \neq 0, m+n}}^{i=m, j=n} a_{i j}(\ln w)_{i, j}+a_{00}
$$

where $u=u(x, t), w=w(x, t),(\ln w)_{i, j}=\partial^{i+j}(\ln w(x, t)) / \partial x^{i}$ $\partial t^{j}, m, n$ (balance numbers), and $a_{i j}(i=0,1, \cdots, m, j=0,1$, $\cdots, n$ ) (balance coefficients) are constants to be determined later. By balancing the highest nonlinear terms and the highest order partial derivative terms, balance numbers are obtained. Substituting Equation (2) into Equation (1) and balancing the terms with $\left(w_{x} / w\right)^{i}\left(w_{t} / w\right)^{j}$ yield a set of algebraic equations for $a_{i j}(i=1, \cdots, m, j=1, \cdots, n)$.

Step 2. Solving the set of algebraic equations and simplifying Equation (1), we can get the bilinear equation of Equation (1) directly or after integrating some times (Generally, integrating times equal to the orders of the lowest partial derivative of Equation (1).) with respect to $x, t$.

Step 3. Generally, in order to obtain the exact solutions of Equation (1), there are three methods to deal with the bilinear equation of Equation (1):

(i) Applying the perturbation method to the bilinear equation of Equation (1), N-soliton solution of Equation (1) can be obtained.

(ii) By using traveling wave transformations

$$
w(x, t)=w(\xi), \quad \xi=x-V t,
$$

the bilinear equation of Equation (1) satisfies the following ODE:

$$
w^{\prime \prime}+\lambda w^{\prime}+\mu w=0
$$

where the prime denotes the derivation with respect to $\xi$ and $\lambda, \mu$, and $V$ are constants to be determined later.

Substituting Equations (3) and (4) into Equation (1), it is converted into the following equation:

$$
l_{1} w^{2}+l_{2} w w^{\prime}+l_{3} w^{\prime 2}=0
$$

where $l_{1}, l_{2}$, and $l_{3}$ are polynomial functions of $V, \lambda$, and $\mu$.

Setting $l_{1}=l_{2}=l_{3}=0$ yields a set of algebraic equations for $V, \lambda$, and $\mu$. Solving the set of algebraic equations and using the solutions of Equation (4), $w$ can be determined. Substituting $w$ into Equation (2), the exact traveling wave solutions of Equation (1) are obtained.

(iii) By applying the compatible condition $\left(w_{x t}=w_{t x}\right)$ to the bilinear equation of Equation (1), it is reduced to an ODE. Solving the ODE, more exact solutions of Equation (1) can be obtained.

Next, we choose the KdV equation and Burgers equation to illustrate our method.

\section{Application to the KdV Equation}

Let us consider the celebrated $\mathrm{KdV}$ equation in the form

$$
u_{t}+u u_{x}+\delta u_{x x x}=0
$$

where $\delta$ is a constant. Suppose that the solution of Equation (6) is of the form

$$
u=a_{m n}(\ln w)_{m, n}+\sum_{\substack{i, j=0 \\ i+j \neq 0, m+n}}^{i=m, j=n} a_{i j}(\ln w)_{i, j}+a_{00}
$$

where $m, n$, and $a_{i j}(i=0,1, \cdots, m, j=0,1, \cdots, n)$ are constants to be determined later.

Balancing $u_{x x x}$ and $u u_{x}$ in Equation (6), it is required that $m+3=2 m+1$ and $n=2 n$. Then, Equation (7) can be written as

$$
u=a_{20}(\ln w)_{x x}+a_{10}(\ln w)_{x}+a_{00}
$$

From Equation (8), one can calculate the following derivatives:

$$
u=a_{20}\left(\frac{w_{x x}}{w}-\frac{w_{x}^{2}}{w^{2}}\right)+a_{10} \frac{w_{x}}{w}+a_{00}
$$

$$
\begin{aligned}
u_{x}= & a_{20}\left(\frac{w_{x x x}}{w}-\frac{3 w_{x x} w_{x}}{w^{2}}+\frac{2 w_{x}^{3}}{w^{3}}\right)+a_{10}\left(\frac{w_{x x}}{w}-\frac{w_{x}^{2}}{w^{2}}\right) \\
u_{t}= & a_{20}\left(\frac{w_{x x t}}{w}-\frac{w_{x x} w_{t}+2 w_{x} w_{x t}}{w^{2}}+2 \frac{w_{x}^{2} w_{t}}{w^{3}}\right) \\
& +a_{10}\left(\frac{w_{x t}}{w}-\frac{w_{x} w_{t}}{w^{2}}\right)
\end{aligned}
$$




$$
\begin{aligned}
u_{x x x}= & a_{20}\left(\frac{w_{x x x x x}}{w}-\frac{5 w_{x x x x} w_{x}+10 w_{x x x} w_{x x}}{w^{2}}\right. \\
& \left.+\frac{20 w_{x x x} w_{x}^{2}+30 w_{x x}^{2} w_{x}}{w^{3}}-\frac{60 w_{x x} w_{x}^{3}}{w^{4}}+\frac{24 w_{x}^{5}}{w^{5}}\right) \\
& +a_{10}\left(\frac{w_{x x x x}}{w}-\frac{4 w_{x x x} w_{x}+3 w_{x x}^{2}}{w^{2}}+\frac{12 w_{x x} w_{x}^{2}}{w^{3}}-\frac{6 w_{x}^{4}}{w^{4}}\right)
\end{aligned}
$$

Equating the coefficients of $\left(w_{x} / w\right)^{5}$ and $\left(w_{x} / w\right)^{4}$ on the left-hand side of Equation (6) to zero yields a set of algebraic equations for $a_{20}$ and $a_{10}$ as follows:

$$
\begin{aligned}
& -2 a_{20}^{2}+24 \delta a_{20}=0, \\
& 3 a_{20} a_{10}-6 \delta a_{10}=0 .
\end{aligned}
$$

Solving the above algebraic equations, we get $a_{20}=12 \delta$ and $a_{10}=0$. Substituting $a_{20}$ and $a_{10}$ back into Equation (8), we get

$$
u=12 \delta(\ln w)_{x x}+a_{00}
$$

where $a_{00}$ is an arbitrary constant. Substituting Equation (11) into Equation (6), we get

$$
12 \delta\left(K_{1}+K_{2}+K_{3}\right)=0
$$

where

$$
\begin{aligned}
K_{1}= & \frac{w_{x x t}}{w}-\frac{2 w_{x} w_{x t}+w_{x x} w_{t}}{w^{2}}+\frac{2 w_{x}^{2} w_{t}}{w^{3}}, \\
K_{2}= & a_{00}\left(\frac{w_{x x x}}{w}-\frac{3 w_{x x} w_{x}}{w^{2}}+\frac{2 w_{x}^{3}}{w^{3}}\right), \\
K_{3}= & \delta\left(\frac{w_{x x x x x}}{w}+\frac{2 w_{x x x} w_{x x}-5 w_{x x x x} w_{x}}{w^{2}}\right. \\
& \left.+\frac{16 w_{x x x} w_{x}^{2}-6 w_{x} w_{x x}^{2}}{w^{3}}\right) .
\end{aligned}
$$

Simplifying Equation (12) and integrating once with respect to $x$, we get

$$
\frac{\partial}{\partial x}\left(\frac{\left(w_{x t} w-w_{x} w_{t}\right)+\delta\left(w_{x x x x} w-4 w_{x} w_{x x x}+3 w_{x x}^{2}\right)+a_{00}\left(w_{x x} w-w_{x}^{2}\right)}{w^{2}}\right)=0
$$

Equation (14) is identical to

$$
\begin{aligned}
& \left(w_{x t} w-w_{x} w_{t}\right)+\delta\left(w_{x x x x} w-4 w_{x} w_{x x x}+3 w_{x x}^{2}\right) \\
& +a_{00}\left(w_{x x} w-w_{x}^{2}\right)-C(t) w^{2}=0
\end{aligned}
$$

where $C(t)$ is an arbitrary function of $t$ and $a_{00}$ is an arbitrary constant.

In particular, taking $C(t)$ as zero in Equation (15), we get the bilinear equation of Equation (6) as follows:

$$
\begin{aligned}
& \left(w_{x t} w-w_{x} w_{t}\right)+\delta\left(w_{x x x x} w-4 w_{x} w_{x x x}+3 w_{x x}^{2}\right) \\
& +a_{00}\left(w_{x x} w-w_{x}^{2}\right)=0 .
\end{aligned}
$$

Equation (16) can be written concisely in terms of $D$ operators as

$$
\left(D_{x} D_{t}+\delta D_{x}^{4}+a_{00} D_{x}^{2}\right) w \cdot w=0,
$$

where

$$
D_{x}^{m} D_{t}^{n} a \cdot b=\left.\left(\partial_{x}-\partial_{x^{\prime}}\right)^{m}\left(\partial_{t}-\partial_{t^{\prime}}\right)^{n} a(x, t) b\left(x^{\prime}, t^{\prime}\right)\right|_{x^{\prime}=x, t^{\prime}=t} .
$$

Remark 1. Applying Hirota's method [22-24], the bilinear equation of Equation (6) can be written as

$$
\left(D_{x} D_{t}+\delta D_{x}^{4}\right) w \cdot w=0
$$

Equation (19) is obtained by setting $a_{00}=0$ in Equation (17). Obviously, Equation (19) is a special case of Equation (17).

(i) Now, we apply the perturbation method to Equation (17) to derive $N$-soliton solution of Equation (6). Suppose that $w$ can be expanded as follows:

$$
w=1+\varepsilon w_{1}+\varepsilon^{2} w_{2}+\cdots \varepsilon^{N} w_{N}+\cdots
$$

where $\varepsilon$ is a parameter and $w_{i}=w_{i}(x, t)(i=1,2, \cdots)$.

Substituting Equation (20) into Equation (17) and arranging it at each order of $\varepsilon$, we get

$$
\begin{aligned}
& \varepsilon: D_{x}\left(D_{t}+a_{00} D_{x}+\delta D_{x}^{3}\right)\left(w_{1} \cdot 1+1 \cdot w_{1}\right)=0, \\
& \varepsilon^{2}: D_{x}\left(D_{t}+a_{00} D_{x}+\delta D_{x}^{3}\right)\left(w_{2} \cdot 1+w_{1} \cdot w_{1}+1 \cdot w_{2}\right)=0, \\
& \varepsilon^{3}: D_{x}\left(D_{t}+a_{00} D_{x}+\delta D_{x}^{3}\right)\left(w_{3} \cdot 1+w_{2} \cdot w_{1}\right. \\
&\left.+w_{1} \cdot w_{2}+1 \cdot w_{3}\right)=0,
\end{aligned}
$$


The order- $\varepsilon$ equation can be rewritten as a linear differential equation for $w_{1}$ as follows:

$$
\frac{\partial}{\partial x}\left(\frac{\partial}{\partial t}+a_{00} \frac{\partial}{\partial x}+\delta \frac{\partial^{3}}{\partial x^{3}}\right) w_{1}=0
$$

Solving Equation (22), we get

$$
w_{1}=e^{P_{1} x-\left(a_{00} P_{1}+\delta P_{1}^{3}\right) t},
$$

where $P_{1}$ is an arbitrary constant.

The coefficient of $\varepsilon^{2}$ can be rearranged as follows:

$$
\begin{aligned}
& 2 \frac{\partial}{\partial x}\left(\frac{\partial}{\partial t}+a_{00} \frac{\partial}{\partial x}+\delta \frac{\partial^{3}}{\partial x^{3}}\right) w_{2} \\
& \quad=-D_{x}\left(D_{t}+a_{00} D_{x}+\delta D_{x}^{3}\right) w_{1} \cdot w_{1}
\end{aligned}
$$

Substituting Equation (23) into Equation (24), the righthand side of Equation (24) equals zero. Therefore, we can choose

$$
w_{2}=0
$$

Substituting Equations (23) and (25) into Equation (20), we get

$$
w=1+e^{P_{1} x-\left(a_{00} P_{1}+\delta P_{1}^{3}\right) t+\xi_{1}^{0}}
$$

where $P_{1}, a_{00}$, and $\xi_{1}^{0}$ are arbitrary constants.

Substituting Equation (26) into Equation (11), 1-soliton solution of Equation (6) can be obtained. If we choose $w_{1}=$ $e^{P_{1} x-\left(a_{00} P_{1}+\delta P_{1}^{3}\right) t}+e^{P_{2} x-\left(a_{00} P_{2}+\delta P_{2}^{3}\right) t}$ in Equation (22), similar to the process of obtaining 1-soliton solution, we can get 2soliton solution of Equation (6) as follows:

$$
w=1+e^{\eta_{1}}+e^{\eta_{2}}+\frac{\left(P_{1}-P_{2}\right)^{2}}{\left(P_{1}-P_{2}\right)^{2}} e^{\eta_{1}+\eta_{2}},
$$

where $\quad \eta_{1}=P_{1} x-\left(a_{00} P_{1}+\delta P_{1}^{3}\right) t+\xi_{1}^{0}, \quad \eta_{2}=P_{2} x-\left(a_{00} P_{2}+\right.$ $\left.\delta P_{2}^{3}\right) t+\xi_{2}^{0}, \quad P_{i}, \xi_{i}^{0}(i=1,2)$, and $a_{00}$ are arbitrary constants.

Substituting Equation (27) into Equation (11), 2-soliton solution of Equation (6) can be obtained. Similarly, we can get $N$-soliton solution of Equation (6).

Remark 2. Obviously, setting $a_{00}=0$ in Equations (23) and (27), 1-soliton and 2-soliton solutions of Equation (6) are identical to Hirota's results [22-24].

Remark 3. By using the properties of D-operators [22-24], a Bäcklund transformation of Equation (17) can be obtained as follows:

$$
\begin{array}{r}
\left(D_{t}+\left(a_{00}+\alpha\right) D_{x}+\delta D_{x}^{3}\right) w^{*} \cdot w=0, \\
\left(D_{x}^{2}-\beta D_{x}-\alpha\right) w^{*} \cdot w=0,
\end{array}
$$

where $w^{*}$ and $w$ satisfy Equation (17) and $\alpha, \beta$, and $a_{00}$ are arbitrary constants.

(ii) Now, we discuss Equation (16) by using the sub-ODE method.

Using transformations $w(x, t)=w(\xi), \xi=x-V t$, Equation (16) is reduced to

$$
\left(a_{00}-V\right)\left(w^{\prime \prime} w-w^{\prime 2}\right)+\delta\left(w^{\prime \prime \prime \prime} w-4 w^{\prime} w^{\prime \prime \prime}+3 w^{\prime \prime 2}\right)=0,
$$

where the prime denotes the derivation with respect to $\xi$ and $V$ is a constant to be determined later.

Noticing the bilinear property of Equation (16), suppose that $w$ satisfies the following ODE:

$$
w^{\prime \prime}+\lambda w^{\prime}+\mu w=0
$$

where $\lambda$ and $\mu$ are parameters.

Substituting Equation (30) into Equation (16), we get

$$
l_{1} w^{2}+l_{2} w w^{\prime}+l_{3} w^{\prime 2}=0
$$

where

$$
\begin{aligned}
& l_{1}=\mu\left(V-a_{00}+\delta\left(4 \mu-\lambda^{2}\right)\right), \\
& l_{2}=\lambda\left(V-a_{00}+\delta\left(4 \mu-\lambda^{2}\right)\right), \\
& l_{3}=V-a_{00}+\delta\left(4 \mu-\lambda^{2}\right) .
\end{aligned}
$$

Setting $l_{1}=l_{2}=l_{3}=0$ yields a set of algebraic equations for $V, \lambda$, and $\mu$. Solving this set of algebraic equations, we get

$$
V=a_{00}+\delta\left(\lambda^{2}-4 \mu\right)
$$

where $\lambda, \mu$, and $a_{00}$ are arbitrary constants.

Substituting Equation (30) into Equation (11), we get

$$
u=12 \delta\left(\frac{w_{x}}{w}+\frac{\lambda}{2}\right)^{2}+3 \delta\left(\lambda^{2}-4 \mu\right)+a_{00}
$$

Substituting the general solutions of Equation (30) into Equation (34), we get three types of traveling wave solutions of Equation (6) as follows.

When $\lambda^{2}-4 \mu>0$,

$$
\begin{aligned}
u_{1}(x, t)= & u_{1}(\xi)=-3 \delta A\left(\frac{C_{1} e^{(\sqrt{A} / 2) \xi}-C_{2} e^{-(\sqrt{A} / 2) \xi}}{C_{1} e^{(\sqrt{A} / 2) \xi}-C_{2} e^{-(\sqrt{A} / 2) \xi}}\right)^{2} \\
& +3 \delta A+a_{00},
\end{aligned}
$$


where

$$
\begin{aligned}
& A=\lambda^{2}-4 \mu, \\
& V=a_{00}+A \delta, \\
& \xi=x-\left(a_{00}+A \delta\right) t,
\end{aligned}
$$

and $\lambda, \mu, C_{1}, C_{2}$, and $a_{00}$ are arbitrary constants.

Taking $C_{1}=C_{3}+C_{4} / 2$ and $C_{2}=C_{3}-C_{4} / 2$, Equation (35) can be rewritten as

$$
\begin{aligned}
u_{2}(x, t)= & u_{2}(\xi) \\
= & -3 \delta A\left(\frac{C_{3} \sinh (\sqrt{A} / 2) \xi+C_{4} \cosh (\sqrt{A} / 2) \xi}{C_{3} \cosh (\sqrt{A} / 2) \xi+C_{4} \sinh (\sqrt{A} / 2) \xi}\right)^{2} \\
& +3 \delta A+a_{00},
\end{aligned}
$$

where $C_{3}, C_{4}$, and $a_{00}$ are arbitrary constants and $A, V$, and $\xi$ are given by Equation (36).

to

In particular, if $\left|C_{4} / C_{3}\right|<1$, then Equation (37) is reduced

$$
u_{3}(x, t)=u_{3}(\xi)=3 \delta A \operatorname{sech}^{2}\left(\frac{\sqrt{A}}{2} \xi+\xi_{0}\right)+a_{00},
$$

where $C_{3}, C_{4}$, and $a_{00}$ are arbitrary constants and $A, V$, and $\xi$ are given by Equation (36), $\xi_{0}=\operatorname{arctanh}\left(C_{4} / C_{3}\right)$.

When $\lambda^{2}-4 \mu<0$,

$$
\begin{aligned}
u_{4}(x, t)= & u_{4}(\xi) \\
= & 3 \delta A\left(\frac{-C_{1} \sin (\sqrt{-A} / 2) \xi+C_{2} \cos (\sqrt{-A} / 2) \xi}{C_{1} \cos (\sqrt{-A} / 2) \xi+C_{2} \sin (\sqrt{-A} / 2) \xi}\right)^{2} \\
& +3 \delta A+a_{00}
\end{aligned}
$$

where $C_{1}, C_{2}$, and $a_{00}$ are arbitrary constants and $A, V$, and $\xi$ are given by Equation (36).

Obviously, Equation (39) can be written as

$$
u_{5}(x, t)=u_{5}(\xi)=3 \delta A \sec ^{2}\left(\frac{\sqrt{-A}}{2} \xi+\xi_{0}\right)+a_{00}
$$

where $C_{1}, C_{2}$, and $a_{00}$ are arbitrary constants and $A, V$, and $\xi$ are given by Equation (36), $\xi_{0}=-\arctan \left(C_{2} / C_{1}\right)$.

When $\lambda^{2}-4 \mu=0$,

$$
u_{6}(x, t)=u_{6}(\xi)=-12 \delta\left(\frac{C_{2}}{C_{1}+C_{2} \xi}\right)^{2}+a_{00}
$$

where $V=a_{00}, \xi=x-a_{00} t, C_{1}, C_{2}$, and $a_{00}$ are arbitrary constants. (iii) Now, we discuss Equation (16) from the compatible condition. Equation (16) can be written as

$$
\begin{aligned}
& \left(\delta w_{x x x x}+w_{x t}+a_{00} w_{x x}\right) w \\
& \quad+\left(\delta\left(3 w_{x x}^{2}-4 w_{x x x} w_{x}\right)-a_{00} w_{x}^{2}-w_{x} w_{t}\right)=0 .
\end{aligned}
$$

Notice $w_{x} \neq 0$; otherwise, we can only get a trivial solution. Setting the second term of Equation (42) to zero and solving $w_{t}$ yield

$$
w_{t}=\frac{\delta\left(3 w_{x x}^{2}-4 w_{x x x} w_{x}\right)-a_{00} w_{x}^{2}}{w_{x}} .
$$

Substituting Equation (43) into Equation (42), we get

$$
w_{x x x x}+\frac{w_{x x}^{3}-2 w_{x} w_{x x} w_{x x x}}{w_{x}^{2}}=0
$$

Integrating Equation (44) once with respect to $x$, we get

$$
w_{x x x}-\frac{w_{x x}^{2}}{w_{x}}=b(t)
$$

where $b(t)$ is an arbitrary function of $t$.

Using transformations $Y=w_{x x}^{2}$ and $X=w_{x}$, Equation (45) is reduced to

$$
\frac{d Y}{d X}=\frac{2 Y}{X}+2 b(t)
$$

Solving the above equation, we get

$$
Y=c(t) X^{2}-2 b(t) X,
$$

namely,

$$
w_{x x}=\sqrt{c(t) w_{x}^{2}-2 b(t) w_{x}}
$$

where $b(t)$ and $c(t)$ are arbitrary functions of $t$.

Case 1. When $b(t)=c(t)=0$, from Equation (48), we get

$$
w=c_{1}(t) x+c_{2}(t)
$$

where $c_{1}(t)$ and $c_{2}(t)$ are arbitrary functions of $t$.

Substituting the above equation into Equation (48), we get

$$
x \frac{d c_{1}(t)}{d t}+\frac{d c_{2}(t)}{d t}=-a_{00} c_{1}(t) .
$$

Setting the coefficients of $x^{i}(i=0,1)$ to zero in the above equation, we get 


$$
\begin{aligned}
& \frac{d c_{1}(t)}{d t}=0, \\
& \frac{d c_{2}(t)}{d t}=-a_{00} c_{1}(t) .
\end{aligned}
$$

Solving the above equations, we get

$$
\begin{aligned}
& c_{1}(t)=C_{1}, \\
& c_{2}(t)=C_{2}-a_{00} C_{1} t,
\end{aligned}
$$

where $C_{i}(i=1,2)$ are arbitrary constants. Then, we get

$$
w=C_{1}\left(x-a_{00} t\right)+C_{2} .
$$

Substituting Equation (53) into Equation (11), we get an exact solution of Equation (6) as follows:

$$
u_{7}(x, t)=a_{00}-\frac{12 \delta C_{1}^{2}}{\left(C_{1}\left(x-a_{00} t\right)+C_{2}\right)^{2}}
$$

where $C_{i}(i=1,2)$ and $a_{00}$ are arbitrary constants.
Case 2. When $b(t)=0$ and $c(t)>0$, similar to Case 1 , we get

$$
w=C_{3}+C_{2} e^{C_{1}\left(x-\left(a_{00}+\delta C_{1}^{2}\right) t\right)}
$$

and an exact solution of Equation (6) as follows:

$$
u_{8}(x, t)=\frac{12 \delta C_{1}^{2} C_{2} C_{3} e^{C_{1}\left(x-\left(a_{00}+\delta C_{1}^{2}\right) t\right)}}{\left(C_{3}+C_{2} e^{C_{1}\left(x-\left(a_{00}+\delta C_{1}^{2}\right) t\right)}\right)^{2}}+a_{00}
$$

where $C_{i}(i=1,2,3)$ and $a_{00}$ are arbitrary constants.

Case 3. When $b(t) \neq 0$ and $c(t)=0$ similar to Case 1 , we get

$$
w=C_{1}\left(x-a_{00} t+C_{2}\right)^{3}+12 \delta C_{1} t+C_{3},
$$

and an exact solution of Equation (6) as follows:

$$
u_{9}(x, t)=a_{00}-\frac{36 \delta C_{1}\left(x-a_{00} t+C_{2}\right)\left(C_{1}\left(x-a_{00} t+C_{2}\right)^{3}-24 \delta C_{1} t-2 C_{3}\right)}{\left(C_{1}\left(x-a_{00} t+C_{2}\right)^{3}-12 \delta C_{1} t+C_{3}\right)^{2}},
$$

where $C_{i}(i=1,2,3)$ and $a_{00}$ are arbitrary constants.

Case 4. When $b(t) \neq 0$ and $c(t)>0$, similar to Case 1 , we get

$$
\begin{aligned}
w= & C_{4} e^{C_{1}\left(x-\left(a_{00}+\delta C_{1}^{2}\right) t\right)}+C_{3} e^{-C_{1}\left(x-\left(a_{00}+\delta C_{1}^{2}\right) t\right)} \\
& +C_{2} x-C_{2}\left(a_{00}+3 \delta C_{1}^{2}\right) t+C_{5},
\end{aligned}
$$

and an exact solution of Equation (6) as follows:

$$
u_{10}(x, t)=12 \delta\left(A_{1}-B_{1}\right)+a_{00}
$$

where

$$
\begin{aligned}
& A_{1}=\frac{C_{1}^{2} C_{4} e^{C_{1}\left(x-\left(a_{00}+\delta C_{1}^{2}\right) t\right)}+C_{1}^{2} C_{3} e^{-C_{1}\left(x-\left(a_{00}+\delta C_{1}^{2}\right) t\right)}}{C_{4} e^{C_{1}\left(x-\left(a_{00}+\delta C_{1}^{2}\right) t\right)}+C_{3} e^{-C_{1}\left(x-\left(a_{00}+\delta C_{1}^{2}\right) t\right)}+C_{2} x-C_{2}\left(a_{00}+3 \delta C_{1}^{2}\right) t+C_{5}}, \\
& B_{1}=\frac{\left(C_{1} C_{4} e^{C_{1}\left(x-\left(a_{00}+\delta C_{1}^{2}\right) t\right)}-C_{1} C_{3} e^{-C_{1}\left(x-\left(a_{00}+\delta C_{1}^{2}\right) t\right)}+C_{2}\right)^{2}}{\left(C_{4} e^{C_{1}\left(x-\left(a_{00}+\delta C_{1}^{2}\right) t\right)}+C_{3} e^{-C_{1}\left(x-\left(a_{00}+\delta C_{1}^{2}\right) t\right)}+C_{2} x-C_{2}\left(a_{00}+3 \delta C_{1}^{2}\right) t+C_{5}\right)^{2}},
\end{aligned}
$$

where $C_{2}^{2}+4 C_{1}^{2} C_{3} C_{4}=0, C_{i}(i=1,2,3,4,5)$, and $a_{00}$ are arbitrary constants.

Case 5. When $b(t) \neq 0$ and $c(t)<0$, similar to Case 1 , we get

$$
w=-\frac{C_{1} C_{2} x+C_{1} \cos \left(C_{2}\left(x+\delta C_{2}^{2} t-a_{00} t+C_{3}\right)\right)+3 \delta C_{1} C_{2}^{3} t-a_{00} C_{1} C_{2} t-C_{4} C_{2}^{3}}{C_{2}^{3}}
$$


and an exact solution of Equation (6) as follows:

$$
u_{11}(x, t)=12 \delta\left(A_{2}-B_{2}\right)+a_{00}
$$

where

$$
\begin{aligned}
& A_{2}=\frac{-C_{1} C_{2}^{2} \cos \left(C_{2}\left(x+\delta C_{2}^{2} t-a_{00} t+C_{3}\right)\right)}{C_{1} C_{2} x+C_{1} \cos \left(C_{2}\left(x+\delta C_{2}^{2} t-a_{00} t+C_{3}\right)\right)+3 \delta C_{1} C_{2}^{3} t-C_{1} C_{2} a_{00} t-C_{2}^{3} C_{4}}, \\
& B_{2}=\frac{C_{1}^{2} C_{2}^{2}\left(-1+\sin \left(C_{2}\left(x+\delta C_{2}^{2} t-a_{00} t+C_{3}\right)\right)\right)^{2}}{\left(C_{1} C_{2} x+C_{1} \cos \left(C_{2}\left(x+\delta C_{2}^{2} t-a_{00} t+C_{3}\right)\right)+3 \delta C_{1} C_{2}^{3} t-a_{00} C_{1} C_{2} t-C_{2}^{3} C_{4}\right)^{2}},
\end{aligned}
$$

where $C_{i}(i=1,2,3,4)$ and $a_{00}$ are arbitrary constants.

So far, based on the bilinear equation which is derived by using the $\mathrm{HB}$ of undetermined coefficient method, many exact solutions of the KdV are obtained by applying the perturbation method, sub-ODE method, and compatible condition. Our results can compare with the $\left(G^{\prime} / G\right)$-expansion method, Hirota's method, and HB method [20-30].

\section{Application to the Burgers Equation}

Let us consider the Burgers equation in the form

$$
u_{t}+u u_{x}+\delta u_{x x}=0
$$

where $\delta$ is a constant.

Suppose that the solution of Equation (65) is of the form

$$
u=a_{m n}(\ln w)_{m, n}+\sum_{\substack{i, j=0 \\ i+j \neq 0, m+n}}^{i=m, j=n} a_{i j}(\ln w)_{i, j}+a_{00}
$$

where $m, n$, and $a_{i j}(i=0,1, \cdots, m, j=0,1, \cdots, n)$ are constants to be determined later.

Balancing $u_{x x}$ and $u u_{x}$ in Equation (65), it is required that $m+2=2 m+1$ and $n=2 n$. Then, Equation (66) can be written as

$$
u=a_{10}(\ln w)_{x}+a_{00} .
$$

From Equation (67), one can calculate the following derivatives:

$$
\begin{aligned}
u & =a_{10} \frac{w_{x}}{w}+a_{00}, \\
u_{x} & =a_{10}\left(\frac{w_{x x}}{w}-\frac{w_{x}^{2}}{w^{2}}\right), \\
u_{t} & =a_{10}\left(\frac{w_{x t}}{w}-\frac{w_{x} w_{t}}{w^{2}}\right), \\
u_{x x} & =a_{10}\left(\frac{w_{x x x}}{w}-\frac{3 w_{x x} w_{x}}{w^{2}}+\frac{2 w_{x}^{3}}{w^{3}}\right) .
\end{aligned}
$$

Equating the coefficients of $\left(w_{x} / w\right)^{3}$ on the left-hand side of Equation (65) to zero yields an algebraic equation for $a_{10}$ as follows:

$$
-a_{10}^{2}+2 a_{10} \delta=0
$$

Solving the above algebraic equation, we get $a_{10}=2 \delta$. Substituting $a_{10}$ back into Equation (67), we get

$$
u=2 \delta(\ln w)_{x}+a_{00}
$$

where $a_{00}$ is an arbitrary constant.

Substituting Equation (70) into Equation (65), we get

$$
2 \delta\left(K_{1}+K_{2}+K_{3}\right)=0
$$

where

$$
\begin{aligned}
& K_{1}=\frac{w_{x t} w-w_{x} w_{t}}{w^{2}}, \\
& K_{2}=a_{00}\left(\frac{w_{x x} w-w_{x}^{2}}{w^{2}}\right), \\
& K_{3}=\delta\left(\frac{w w_{x x x}-w_{x} w_{x x}}{w^{2}}\right) .
\end{aligned}
$$

Simplifying Equation (71), we get

$$
\left(w_{x t} w-w_{x} w_{t}\right)+a_{00}\left(w_{x x} w-w_{x}^{2}\right)+\delta\left(w w_{x x x}-w_{x} w_{x x}\right)=0 .
$$

Equation (73) can be written concisely in terms of $D$ operators as

$$
D_{x}\left(w_{t}+\delta w_{x x}+a_{00} w_{x}\right) \cdot w=0 \text {. }
$$

Equation (74) is identical to

$$
w_{t}+\delta w_{x x}+a_{00} w_{x}-C(t) w=0
$$

where $C(t)$ is an arbitrary function of $t$ and $a_{00}$ is an arbitrary constant. 
In particular, taking $C(t)$ as constant $C$ in Equation (75), we get

$$
w_{t}+\delta w_{x x}+a_{00} w_{x}-C w=0 .
$$

Remark 4. Applying Hirota's method [22-24], the bilinear equation of Equation (74) can be written as

$$
D_{x}\left(w_{t}+\delta w_{x x}\right) \cdot w=0 .
$$

Equation (77) is obtained by setting $a_{00}=0$ in Equation (74). Obviously, Equation (77) is a special case of Equation (74).

Remark 5. Equations (70) and (76) are general Cole-Hopf transformations. In fact, setting $a_{00}=C=0$ in Equations (70) and (76), we get the famous Cole-Hopf transformations

$$
u=2 \delta(\ln w)_{x}, w_{t}+\delta w_{x x}=0 .
$$

(i) Now, we apply the perturbation method to Equation (74) to derive $N$-soliton solution of Equation (65). Suppose that $w$ can be expanded as follows:

$$
w=1+\varepsilon w_{1}+\varepsilon^{2} w_{2}+\cdots \varepsilon^{N} w_{N}+\cdots,
$$

where $\varepsilon$ is a parameter and $w_{i}=w_{i}(x, t)(i=1,2, \cdots)$.

Substituting Equation (79) into Equation (74) and arranging it at each order of $\varepsilon$, we get

$$
\begin{aligned}
\varepsilon & : \frac{1}{2}\left(D_{x t}+a_{00} D_{x}^{2}\right)\left(w_{1} \cdot 1+1 \cdot w_{1}\right)+\delta D_{x} w_{1 x x} \cdot 1=0, \\
\varepsilon^{2} & : \frac{1}{2}\left(D_{x t}+a_{00} D_{x}^{2}\right)\left(w_{2} \cdot 1+w_{1} \cdot w_{1}+1 \cdot w_{2}\right) \\
& +\delta D_{x}\left(w_{1 x x} \cdot w_{1}+w_{2 x x} \cdot 1\right)=0, \\
\varepsilon^{3} & : \frac{1}{2}\left(D_{x t}+a_{00} D_{x}^{2}\right)\left(w_{3} \cdot 1+w_{2} \cdot w_{1}+w_{1} \cdot w_{2}+1 \cdot w_{3}\right) \\
\quad & +\delta D_{x}\left(w_{1 x x} \cdot w_{2}+w_{2 x x} \cdot w_{1}+w_{3 x x} \cdot 1\right)=0,
\end{aligned}
$$

The order- $\varepsilon$ equation can be rewritten as a linear differential equation for $w_{1}$ as follows:

$$
\frac{\partial}{\partial x}\left(\frac{\partial}{\partial t}+a_{00} \frac{\partial}{\partial x}+\delta \frac{\partial^{2}}{\partial x^{2}}\right) w_{1}=0
$$

Solving Equation (81), we get

$$
w_{1}=e^{P_{1} x-\left(a_{00} P_{1}+\delta P_{1}^{2}\right) t},
$$

where $P_{1}$ is an arbitrary constant.
The coefficient of $\varepsilon^{2}$ can be rearranged as follows:

$$
\begin{aligned}
& \frac{\partial}{\partial x}\left(\frac{\partial}{\partial t}+a_{00} \frac{\partial}{\partial x}+\delta \frac{\partial^{2}}{\partial x^{2}}\right) w_{2} \\
& \quad=-\frac{1}{2}\left(D_{x t}+a_{00} D_{x}^{2}\right) w_{1} \cdot w_{1}-\delta D_{x} w_{1 x x} \cdot w_{1} .
\end{aligned}
$$

Substituting Equation (82) into Equation (83), the righthand side of Equation (83) equals zero. Therefore, we can choose

$$
w_{2}=0 \text {. }
$$

Substituting Equations (82) and (84) into Equation (79), we get

$$
w=1+e^{P_{1} x-\left(a_{00} P_{1}+\delta P_{1}^{2}\right) t+\xi_{1}^{0}},
$$

where $P_{1}, a_{00}$, and $\xi_{1}{ }^{0}$ are arbitrary constants.

Substituting Equation (85) into Equation (70), 1-soliton solution of Equation (65) can be obtained.

If we choose $w_{1}=e^{P_{1} x-\left(a_{00} P_{1}+\delta P_{1}^{2}\right) t}+e^{P_{2} x-\left(a_{00} P_{2}+\delta P_{2}^{2}\right) t}$ in Equation (81), Equation (74) has no 2-soliton solution. But there exists a solution as follows:

$$
w=1+\sum_{i=1}^{n} e^{\eta_{1}},
$$

where $\eta_{i}=P_{i} x-\left(a_{00} P_{i}+\delta P_{i}^{2}\right) t+\xi_{1}^{0}, P_{i}, \xi_{1}^{0}(i=1,2, \cdots, n)$, and $a_{00}$ are arbitrary constants.

(ii) Now, we discuss Equation (73) by using the sub-ODE method.

Using transformations $w(x, t)=w(\xi), \xi=x-V t$, Equation (73) is reduced to

$$
\left(a_{00}-V\right)\left(w^{\prime \prime} w-w^{\prime 2}\right)+\delta\left(w w^{\prime \prime \prime}-w^{\prime} w^{\prime \prime}\right)=0
$$

where the prime denotes the derivation with respect to $\xi$ and $V$ is a constant to be determined later.

Noticing the bilinear property of Equation (87), suppose that $w$ satisfies the following ODE:

$$
w^{\prime \prime}+\lambda w^{\prime}+\mu w=0
$$

where $\lambda$ and $\mu$ are parameters.

Substituting Equation (88) into Equation (87), we get

$$
l_{1} w^{2}+l_{2} w w^{\prime}+l_{3} w^{\prime 2}=0
$$


where

$$
\begin{aligned}
& l_{1}=\mu\left(\delta \lambda+V-a_{00}\right), \\
& l_{2}=\lambda\left(\delta \lambda+V-a_{00}\right), \\
& l_{3}=\delta \lambda+V-a_{00} .
\end{aligned}
$$

Setting $l_{1}=l_{2}=l_{3}=0$ yields a set of algebraic equations for $V, \lambda$, and $\mu$. Solving the set of algebraic equations, we get

$$
V=a_{00}-\delta \lambda,
$$

where $\lambda, \mu$, and $a_{00}$ are arbitrary constants.

Substituting the general solutions of Equation (88) into Equation (70), we get three types of traveling wave solutions of Equation (65) as follows.

When $\lambda^{2}-4 \mu>0$,

$u_{1}(x, t)=u_{1}(\xi)=\delta \sqrt{A}\left(\frac{C_{1} e^{(\sqrt{A} / 2) \xi}-C_{2} e^{-(\sqrt{A} / 2) \xi}}{C_{1} e^{(\sqrt{A} / 2) \xi}+C_{2} e^{-(\sqrt{A} / 2) \xi}}\right)+a_{00}-\delta \lambda$,

where

$$
\begin{aligned}
& A=\lambda^{2}-4 \mu, \\
& V=a_{00}-\delta \lambda, \\
& \xi=x-\left(a_{00}-\delta \lambda\right) t,
\end{aligned}
$$

where $C_{1}, C_{2}, \lambda, \mu$, and $a_{00}$ are arbitrary constants.

Taking $C_{1}=\left(C_{3}+C_{4}\right) / 2$ and $C_{2}=\left(C_{3}-C_{4}\right) / 2$, Equation (93) can be written as

$$
\begin{aligned}
u_{2}(x, t)= & u_{2}(\xi)=\delta \sqrt{A} \\
& \cdot\left(\frac{C_{3} \sinh (\sqrt{A} / 2) \xi+C_{4} \cosh (\sqrt{A} / 2) \xi}{C_{3} \cosh (\sqrt{A} / 2) \xi+C_{4} \sinh (\sqrt{A} / 2) \xi}\right) \\
& +a_{00}-\delta \lambda,
\end{aligned}
$$

where $C_{3}, C_{4}$, and $a_{00}$ are arbitrary constants and $A$ and $\xi$ are given by Equation (94).

In particular, if $\left|C_{4} / C_{3}\right|<1$, then Equation (94) is reduced to

$$
u_{3}(x, t)=u_{3}(\xi)=\delta \sqrt{A} \tanh \left(\frac{\sqrt{A}}{2} \xi+\xi_{0}\right)+a_{00}-\delta \lambda,
$$

where $C_{3}, C_{4}$, and $a_{00}$ are arbitrary constants and $A$ and $\xi$ are given by Equation (94), $\xi_{0}=\operatorname{arctanh}\left(C_{4} / C_{3}\right)$.
When $\lambda^{2}-4 \mu<0$,

$$
\begin{aligned}
u_{4}(x, t)= & u_{4}(\xi)=\delta \sqrt{-A} \\
& \cdot\left(\frac{-C_{3} \sin (\sqrt{-A} / 2) \xi+C_{4} \cos (\sqrt{-A} / 2) \xi}{C_{3} \cos (\sqrt{-A} / 2) \xi+C_{4} \sin (\sqrt{-A} / 2) \xi}\right) \\
& +a_{00}-\delta \lambda,
\end{aligned}
$$

where $C_{3}, C_{4}$, and $a_{00}$ are arbitrary constants and $A$ and $\xi$ are given by Equation (94).

Obviously, Equation (96) can be written as

$$
u_{5}(x, t)=u_{5}(\xi)=-\delta \sqrt{-A} \tan \left(\frac{\sqrt{-A}}{2} \xi-\xi_{0}\right)+a_{00}-\delta \lambda,
$$

where $\lambda, \mu$, and $a_{00}$ are arbitrary constants and $A$ and $\xi$ are given by Equation (94), $\xi_{0}=-\arctan \left(C_{4} / C_{3}\right)$.

When $\lambda^{2}-4 \mu=0$,

$$
u_{6}(x, t)=u_{6}(\xi)=\frac{2 \delta C^{2}}{C_{1}+C_{2} \xi}+a_{00}-\delta \lambda,
$$

where $\xi=x-\left(a_{00}-\delta \lambda\right) t, C_{1}, C_{2}, \lambda, \mu$, and $a_{00}$ are arbitrary constants.

(iii) Now, we discuss Equation (73) from the compatible condition.

Using the compatible condition, we can get nothing but Equation (76). Using transformations $w(x, t)=w(\xi)$, $\xi=x-V t$, Equation (76) is reduced to

$$
\delta w^{\prime \prime}+\left(a_{00}-V\right) w^{\prime}-C w=0 .
$$

Substituting the general solutions of Equation (99) into Equation (70), we get three types of traveling wave solutions of Equation (65) as follows.

When $\left(a_{00}-V_{1}\right)^{2}+4 C \delta>0$,

$$
u_{7}(x, t)=u_{7}(\xi)=\sqrt{\Delta}\left(\frac{C_{1} e^{(\sqrt{\Delta} / 2 \delta) \xi}-C_{2} e^{-(\sqrt{\Delta} / 2 \delta) \xi}}{C_{1} e^{(\sqrt{\Delta} / 2 \delta) \xi}+C_{2} e^{-(\sqrt{\Delta} / 2 \delta) \xi}}\right)+V_{1},
$$

where

$$
\begin{aligned}
& \Delta=\left(a_{00}-V_{1}\right)^{2}+4 C \delta, \\
& \xi=x-V_{1} t,
\end{aligned}
$$

where $V_{1}, C, C_{1}, C_{2}$, and $a_{00}$ are arbitrary constants. 
Taking $C_{1}=\left(C_{3}+C_{4}\right) / 2$ and $C_{2}=\left(C_{3}+C_{4}\right) / 2$, Equation (101) can be written as

$$
\begin{aligned}
u_{8}(x, t)= & u_{8}(\xi)=\sqrt{\Delta} \\
& \cdot\left(\frac{C_{3} \sinh (\sqrt{\Delta} / 2 \delta) \xi+C_{4} \cosh (\sqrt{\Delta} / 2 \delta) \xi}{C_{3} \cosh (\sqrt{\Delta} / 2 \delta) \xi+C_{4} \sinh (\sqrt{\Delta} / 2 \delta) \xi}\right) \\
& +V_{1},
\end{aligned}
$$

where $V_{1}, C, C_{3}, C_{4}$, and $a_{00}$ are arbitrary constants and $\Delta$ and $\xi$ are given by Equation (101).

In particular, if $C_{4} / C_{3}<1$, then Equation (102) is reduced to

$$
u_{9}(x, t)=u_{9}(\xi)=\sqrt{\Delta} \tanh \left(\frac{\sqrt{\Delta}}{2 \delta} \xi+\xi_{0}\right)+V_{1}
$$

where $\xi_{0}=\operatorname{arctanh}\left(C_{4} / C_{3}\right), V_{1}, C, C_{3}, C_{4}$, and $a_{00}$ are arbitrary constants and $\Delta$ and $\xi$ are given by Equation (101).

$$
\text { When }\left(a_{00}-V_{2}\right)^{2}+4 C \delta<0 \text {, }
$$

$$
\begin{aligned}
u_{10}(x, t)= & u_{10}(\xi)=\sqrt{-\Delta} \\
& \cdot\left(\frac{-C_{3} \sin (\sqrt{-\Delta} / 2 \delta) \xi+C_{4} \cos (\sqrt{-\Delta} / 2 \delta) \xi}{C_{3} \cos (\sqrt{-\Delta} / 2 \delta) \xi+C_{4} \sin (\sqrt{-\Delta} / 2 \delta) \xi}\right) \\
& +V_{2},
\end{aligned}
$$

where $V_{2}, C, C_{3}, C_{4}$, and $a_{00}$ are arbitrary constants and $\Delta$ and $\xi$ are given by Equation (101).

Obviously, Equation (104) can be written as

$$
u_{11}(x, t)=u_{11}(\xi)=-\sqrt{-\Delta} \tan \left(\sqrt{\frac{\sqrt{-\Delta}}{2 \delta}} \xi-\xi_{0}\right)+V_{2}
$$

where $\xi_{0}=-\arctan \left(C_{4} / C_{3}\right), V_{2}, C, C_{3}, C_{4}$, and $a_{00}$ are arbitrary constants and $\Delta$ and $\xi$ are given by Equation (101).

When $\left(a_{00}-V_{3}\right)^{2}+4 C \delta=0$,

$$
u_{12}(x, t)=u_{12}(\xi)=2 \delta \frac{C_{2}}{C_{1}+C_{2} \xi}+V_{3},
$$

where $\xi=x-V_{3} t, V_{3}, C_{1}, C_{2}$, and $a_{00}$ are arbitrary constants.

Moreover, note that Equation (76) is linear, so we can get the solution of Equation (65) as follows:

$$
w=\sum_{i=1}^{n_{1}} w_{1 i}+\sum_{i=1}^{n_{2}} w_{2 i}+\sum_{i=1}^{n_{3}} w_{3 i} u_{13}=2 \delta(\ln w)+a_{00}
$$

where

$$
\begin{aligned}
w_{1 i}= & C_{1,1 i} e^{\left(\left(-\left(a_{00}-V_{1 i}\right)+\sqrt{\Delta_{1 i}}\right) / 2\right) \xi_{1 i}} \\
& +C_{2,1 i} e^{\left(\left(-\left(a_{00}-V_{1 i}\right)-\sqrt{\Delta_{1 i}}\right) / 2\right) \xi_{1 i},} \\
w_{2 i}= & \left(C_{1,2 i} \cos \left(\frac{\sqrt{-\Delta_{2 i}} \xi_{2 i}}{2}\right)\right. \\
& \left.+C_{2,2 i} \sin \left(\frac{\sqrt{-\Delta_{2 i}} \xi_{2 i}}{2}\right)\right) e^{\left(\left(-\left(a_{00}-V_{2 i}\right) \xi_{2 i}\right) / 2\right)}, \\
w_{3 i}= & \left(C_{1,3 i}+C_{2,3 i} \xi_{3 i}\right) e^{\left(\left(-\left(a_{00}-V_{3 i}\right) \xi_{3 i}\right) / 2\right),}
\end{aligned}
$$

where $\quad \xi_{1 i}=x-V_{1 i} t, \quad \Delta_{1 i}=\left(a_{00}-V_{1 i}\right)^{2}+4 C \delta>0$ $\left(i=1, \cdots, n_{1}\right) ; \quad \xi_{2 i}=x-V_{2 i} t, \quad \Delta_{2 i}=\left(a_{00}-V_{2 i}\right)^{2}+4 C \delta<0$ $\left(i=1, \cdots, n_{2}\right) ; \quad \xi_{3 i}=x-V_{3 i} t, \quad \Delta_{3 i}=\left(a_{00}-V_{3 i}\right)^{2}+4 C \delta=0$ $\left(i=1, \cdots, n_{3}\right) ; C_{1,1 i}, C_{2,1 i}\left(i=1, \cdots, n_{1}\right) ; C_{1,2 i}, C_{2,2 i}(i=1, \cdots$, $\left.n_{2}\right)$; and $C_{1,3 i}, C_{2,3 i}\left(i=1, \cdots, n_{3}\right)$ are arbitrary constants, and $n_{1}, n_{2}$, and $n_{3}$ are arbitrary but finite integers.

Remark 6. We can deal with Equation (76) by using some assumptions. For example, when we suppose that $w=-$ $\alpha t+W(x)$ and $C=0$, we get

$$
\begin{gathered}
w=-\alpha t+\frac{\alpha x}{a_{00}}+C_{1}+C_{2} \mathrm{e}^{-a_{00} x / \delta}, \\
u_{14}(x, t)=\frac{\alpha\left(2 \delta-t a_{00}^{2}+a_{00} x\right)+a_{00}^{2}\left(C_{1}-C_{2} e^{-a_{00} x / \delta}\right)}{\alpha\left(x-a_{00} t\right)+a_{00}\left(C_{1}+C_{2} e^{-a_{00} x / \delta}\right)},
\end{gathered}
$$

where $C_{1}, C_{2}, \alpha$, and $a_{00}$ are arbitrary constants.

When we suppose that $w=-\alpha t+W(x)$ and $C=a_{00}=0$, we get

$$
\begin{aligned}
w & =-\alpha t+\frac{\alpha x^{2}}{2 \delta}+C_{1} x+C_{2}, \\
u_{15}(x, t) & =\frac{4 \delta\left(\alpha x+\delta C_{1}\right)}{2 \delta\left(C_{1} x+C_{2}-\alpha t\right)+\alpha x^{2}},
\end{aligned}
$$

where $C_{1}, C_{2}$, and $\alpha$ are arbitrary constants.

Similarly, we can assume that $w=\sum_{i=1}^{n} p_{i}(x) q_{i}(t)$; then, a new solution of Equation (65) can be obtained. Being similar to above process, we omit it.

So far, applying the $\mathrm{HB}$ of undetermined coefficient method to the Burgers equation, we get the bilinear equation of Burgers equation. Moreover, we reduce the Burgers to a linear equation. Based on them, many exact solutions of the Burgers equation are obtained by applying the perturbation method, sub-ODE method, and compatible condition. Our results can compare with the $\left(G^{\prime} / G\right)$-expansion method, Hirota's method, and HB method [20-30]. 


\section{Bilinear Equation of the Boussinesq Equation and Sawada-Kotera Equation}

In this section, we derive the bilinear equations of the Boussinesq equation and Sawada-Kotera equation by using the $\mathrm{HB}$ of undetermined coefficient method. Being similar to Section 4, we omit the process of solving exact solutions.

Example 1. The generalized Boussinesq equation reads

$$
u_{t t}+2 \alpha u_{x t}+\left(\alpha^{2}+\beta\right) u_{x x}+\gamma u u_{x x}+\delta u_{x x x x}=0,
$$

where $\alpha, \beta, \gamma$, and $\delta$ are known constants.

In order to balance $u_{x x x x}$ and $u u_{x}$ in Equation (111), it is required that $m+4=2 m+2$ and $n=2 n$. Then, we suppose that the solution of Equation (111) is of the form

$$
u=a_{20}(\ln w)_{x x}+a_{10}(\ln w)_{x}+a_{00}
$$

where $a_{i 0}(i=0,1,2)$ are constants to be determined later.

Substituting Equation (112) into Equation (111) and equating the coefficients of $\left(w_{x} / w\right)^{6}$ and $\left(w_{x} / w\right)^{5}$ on the left-hand side of Equation (111) to zero yield a set of algebraic equations for $a_{20}$ and $a_{10}$. Solving the algebraic equations, we get $a_{20}=6 \delta / \gamma$ and $a_{10}=0$. Substituting $a_{20}$ and $a_{10}$ back into Equation (112), we get

$$
u=\frac{6 \delta}{\gamma}(\ln w)_{x x}+a_{00} .
$$

where $a_{00}$ is an arbitrary constant.

Substituting Equation (113) into Equation (111), we get

$$
\frac{6 \delta}{\gamma}\left(K_{1}+K_{2}+K_{3}\right)=0,
$$

where

$$
\begin{aligned}
K_{1}= & \beta\left(\frac{w_{x x x x}}{w}-\frac{3 w_{x x}^{2}+4 w_{x} w_{x x x}}{w^{2}}+\frac{12 w_{x x} w_{x}^{2}}{w^{3}}-\frac{6 w_{x}^{4}}{w^{4}}\right) \\
& +\alpha^{2}\left(\frac{12 w_{x x} w_{x}^{2}}{w^{3}}-\frac{3 w_{x x}^{2}+4 w_{x} w_{x x x}}{w^{2}}+\frac{w_{x x x x}}{w}-\frac{6 w_{x}^{4}}{w^{4}}\right) \\
& +\alpha\left(\frac{2 w_{x x x t}}{w}-\frac{6 w_{x x} w_{x t}+6 w_{x x t} w_{x}+2 w_{t} w_{x x x}}{w^{2}}\right. \\
& \left.+\frac{12 w_{x} w_{t} w_{x x}+12 w_{x}^{2} w_{x t}}{w^{3}}-\frac{12 w_{t} w_{x}^{3}}{w^{4}}\right)+\frac{w_{x x t t}}{w} \\
& -\frac{2 w_{x} w_{x t t}+w_{x x} w_{t t}+2 w_{t} w_{x x t}+2 w_{x t}^{2}}{w^{2}} \\
& +\frac{2 w_{x}^{2} w_{t t}+2 w_{x x} w_{t}^{2}+8 w_{x} w_{t} w_{x t}}{w^{3}}-\frac{6 w_{x}^{2} w_{t}^{2}}{w^{4}} \\
K_{2}= & a_{00}\left(\frac{2 \gamma w_{x x x x}}{w}-\frac{8 \gamma w_{x} w_{x x x}+6 \gamma w_{x x}^{2}}{w^{2}}\right. \\
& \left.+\frac{24 \gamma w_{x x} w_{x}^{2}}{w^{3}}-\frac{12 \gamma w_{x}^{4}}{w^{4}}\right),
\end{aligned}
$$

$$
\begin{aligned}
K_{3}= & \delta\left(\frac{w_{x x x x x x}}{w}+\frac{2 w_{x x x}^{2}-6 w_{x} w_{x x x x x}-3 w_{x x} w_{x x x x}}{w^{2}}\right. \\
& \left.+\frac{18 w_{x}^{2} w_{x x x x}-6 w_{x x}^{3}}{w^{3}}+\frac{18 w_{x}^{2} w_{x x}^{2}-24 w_{x}^{3} w_{x x x}}{w^{4}}\right) .
\end{aligned}
$$

Simplifying Equation (114) and integrating twice with respect to $x$, we get

$$
\begin{gathered}
\frac{\partial^{2}}{\partial x^{2}}\left(\left(\alpha^{2}+\beta+2 \gamma a_{00}\right)\left(\frac{w w_{x x}-w_{x}^{2}}{w^{2}}\right)+2 \alpha\left(\frac{w w_{x t}-w_{x} w_{t}}{w^{2}}\right)\right. \\
\left.+\left(\frac{w w_{t t}-w_{t}^{2}}{w^{2}}\right)+\delta\left(\frac{w w_{x x x x}-4 w_{x} w_{x x x} 3 w_{x x}^{2}}{w^{2}}\right)\right)=0 .
\end{gathered}
$$

Equation (116) is identical to

$$
\begin{aligned}
& \left(\alpha^{2}+\beta+2 \gamma a_{00}\right)\left(w w_{x x}-w_{x}^{2}\right)+2 \alpha\left(w w_{x t}-w_{x} w_{t}\right) \\
& \quad+\left(w w_{t t}-w_{t}^{2}\right)+\delta\left(w w_{x x x x}-4 w_{x} w_{x x x}+3 w_{x x}^{2}\right) \\
& \quad-\left(C_{1}(t) x+C_{2}(t)\right) w^{2}=0
\end{aligned}
$$

where $C_{1}(t)$ and $C_{2}(t)$ are arbitrary functions of $t$ and $a_{00}$ is an arbitrary constant.

In particular, letting $C_{1}(t)=C_{2}(t)=0$ in Equation (117), we get the bilinear equation of Equation (111) as follows:

$$
\begin{aligned}
& \left(\alpha^{2}+\beta+2 \gamma a_{00}\right)\left(w w_{x x}-w_{x}^{2}\right)+2 \alpha\left(w w_{x t}-w_{x} w_{t}\right) \\
& \quad+\left(w w_{t t}-w_{t}^{2}\right)+\delta\left(w w_{x x x x}-4 w_{x} w_{x x x}+3 w_{x x}^{2}\right)=0
\end{aligned}
$$

Equation (118) can be written concisely in terms of $D$ operators as

$$
\left(2 \alpha D_{x} D_{t}+\delta D_{x}^{4}+\left(\alpha^{2}+\beta+2 \gamma a_{00}\right) D_{x}^{2}+D_{t}^{2}\right) w \cdot w=0
$$

where $a_{00}$ is an arbitrary constant.

Example 2. The Sawada-Kotera equation reads

$$
u_{t}+15\left(u^{3}+u u_{x x}\right)_{x}+u_{x x x x x}=0
$$

In order to balance $u_{x x x x x}$ and $u^{3}$ in Equation (120), it is required that $m+5=3 m+1$ and $n=3 n$. Then, we suppose that the solution of Equation (120) is of the form

$$
u=a_{20}(\ln w)_{x x}+a_{10}(\ln w)_{x}+a_{00}
$$

where $a_{i 0}(i=0,1,2)$ are constants to be determined later.

Substituting Equation (121) into Equation (120) and equating the coefficients of $\left(w_{x} / w\right)^{7}$ and $\left(w_{x} / w\right)^{6}$ on the left-hand side of Equation (120) to zero yield a set of algebraic equations for $a_{20}$ and $a_{10}$. Solving the algebraic 
equations, we get $a_{20}=2$ and $a_{10}=0$. Substituting $a_{20}$ and $a_{10}$ back into Equation (121), we get

$$
u=2(\ln w)_{x x}+a_{00}
$$

where $a_{00}$ is an arbitrary constant.

Substituting Equation (122) into Equation (120), we get

$$
2\left(K_{1}+K_{2}+K_{3}\right)=0
$$

where

$$
\begin{aligned}
K_{1}= & 45 a_{00}^{2}\left(\frac{w_{x x x}}{w}-\frac{3 w_{x} w_{x x}}{w^{2}}+\frac{2 w_{x}^{3}}{w^{3}}\right) \\
K_{2}= & 15 a_{00}\left(\frac{w_{x x x x x}}{w}+\frac{2 w_{x x} w_{x x x}-5 w_{x} w_{x x x x}}{w^{2}}\right. \\
& \left.+\frac{\left.8 w_{x x x} w_{x}^{2}-6 w_{x} w_{x x}^{2}\right)}{w^{3}}\right), \\
K_{3}= & \frac{\left.w_{x x t}-\frac{2 w_{x} w_{x t}+w_{x x} w_{t}}{w^{2}}+\frac{2 w_{x}^{2} w_{t}}{w^{3}}\right)}{}+\left(\frac{w_{x x x x x x x}}{w}+\frac{9 w_{x x} w_{x x x x x}-5 w_{x x x} w_{x x x x}-7 w_{x} w_{x x x x x x}}{w^{2}}\right. \\
& \left.+\frac{20 w_{x} w_{x x x}^{2}-30 w_{x} w_{x x} w_{x x x x}+12 w_{x}^{2} w_{x x x x x}}{w^{3}}\right) .
\end{aligned}
$$

Simplifying Equation (123) and integrating once with respect to $x$, we get

$$
\begin{aligned}
& \frac{\partial}{\partial x}\left(45 a_{00}^{2}\left(\frac{w w_{x x}-w_{x}^{2}}{w^{2}}\right)\right. \\
& +15 a_{00}\left(\frac{w w_{x x x x}-4 w_{x} w_{x x x}+3 w_{x x}^{2}}{w^{2}}\right)+\frac{w w_{x t}-w_{x} w_{t}}{w^{2}} \\
& \left.+\frac{w w_{x x x x x x}-6 w_{x} w_{x x x x x}+15 w_{x x} w_{x x x x}-10 w_{x x x}^{2}}{w^{2}}\right)=0 .
\end{aligned}
$$

Equation (125) is identical to

$$
\begin{aligned}
& 45 a_{00}^{2}\left(w w_{x x}-w_{x}^{2}\right)+15 a_{00}\left(w w_{x x x x}-4 w_{x} w_{x x x}+3 w_{x x}^{2}\right) \\
& \quad+\left(w w_{x t}-w_{x} w_{t}\right)+\left(w w_{x x x x x x}-6 w_{x} w_{x x x x x}\right. \\
& \left.\quad+15 w_{x x} w_{x x x x}-10 w_{x x x}^{2}\right)-C(t) w^{2}=0
\end{aligned}
$$

where $C(t)$ is an arbitrary function of $t$ and $a_{00}$ is an arbitrary constant.
In particular, letting $C(t)=0$ in Equation (126), we get the bilinear equation of Equation (120) as follows:

$$
\begin{aligned}
& 45 a_{00}^{2}\left(w w_{x x}-w_{x}^{2}\right)+15 a_{00}\left(w w_{x x x x}-4 w_{x} w_{x x x}+3 w_{x x}^{2}\right) \\
& \quad+\left(w w_{x t}-w_{x} w_{t}\right)+\left(w w_{x x x x x x}-6 w_{x} w_{x x x x x}\right. \\
& \left.\quad+15 w_{x x} w_{x x x x}-10 w_{x x x}^{2}\right)=0
\end{aligned}
$$

Equation (127) can be written concisely in terms of $D$ operators as

$$
\left(45 a_{00}^{2} D_{x}^{2}+15 a_{00} D_{x}^{4}+D_{x} D_{t}+D_{x}^{6}\right) w \cdot w=0
$$

where $a_{00}$ is an arbitrary constant.

\section{Conclusions}

The HB of undetermined coefficient method is successfully used to establish the bilinear equation of NLPDE. By applying the perturbation method, sub-ODE method, and compatible condition to the bilinear equation, more exact solutions of NLPDE are obtained. We illustrate the real meaning of balance numbers. We show the underlying relations among the $\left(G^{\prime} / G\right)$-expansion method, Hirota's method, and HB method. Many well-known NLPDE can be handled by the $\mathrm{HB}$ of undetermined coefficient method. The performance of our method is found to be simple and efficient. The availability of computer systems like Maple facilitates the tedious algebraic calculations. Our method is also a standard and computable method, which allows us to solve complicated and tedious algebraic calculations.

\section{Data Availability}

The data used to support the findings of this study are available from the corresponding author upon request.

\section{Conflicts of Interest}

The authors declare no competing interests.

\section{Authors' Contributions}

Xiao-Feng Yang analyzed and interpreted the data and wrote the manuscript; Yi Wei designed and optimized the algorithm and program. All authors read the manuscript.

\section{Acknowledgments}

The research is supported by the Fundamental Research Funds for the Central Universities (2452017373), the Doctoral Research Foundation of Northwest A\&F University (2452017007), the Project of Shandong Province Higher Educational Science and Technology Program (J18KB100), the NSFC Cultivation Project of Jining Medical University (JYP2018KJ15), the Doctoral Research Foundation of Jining Medical University (2017JYQD22). 


\section{References}

[1] X. G. Zhang, L. S. Liu, Y. H. Wu, and Y. J. Cui, "Existence of infinitely solutions for a modified nonlinear Schrödinger equation via dual approach," Electronic Journal of Differential Equations, vol. 2018, no. 147, pp. 1-15, 2018.

[2] C. Chen, X. Zhang, G. Zhang, and Y. Zhang, "A two-grid finite element method for nonlinear parabolic integro-differential equations," International Journal of Computer Mathematics, vol. 96, no. 10, pp. 2010-2023, 2019.

[3] X. Zhang, Y. Wu, and L. Caccetta, "Nonlocal fractional order differential equations with changing-sign singular perturbation," Applied Mathematical Modelling, vol. 39, no. 21, pp. 6543-16552, 2015.

[4] C. Chen, W. Liu, and C. Bi, "A two-grid characteristic finite volume element method for semilinear advection-dominated diffusion equations," Numerical Methods for Partial Differential Equations, vol. 29, no. 5, pp. 1543-1562, 2013.

[5] X. Zhang, L. Liu, Y. Wu, and Y. Cui, "The existence and nonexistence of entire large solutions for a quasilinear Schrödinger elliptic system by dual approach," Journal of Mathematical Analysis and Applications, vol. 464, no. 2, pp. 1089-1106, 2018.

[6] X. Zhang, J. Jiang, Y. Wu, and Y. Cui, "Existence and asymptotic properties of solutions for a nonlinear Schrödinger elliptic equation from geophysical fluid flows," Applied Mathematics Letters, vol. 90, pp. 229-237, 2019.

[7] J. Liu, P. A. Perry, and C. Sulem, "Global existence for the derivative nonlinear Schrödinger equation by the method of inverse scattering," Communications in Partial Differential Equations, vol. 41, no. 11, pp. 1692-1760, 2016.

[8] H. Mao, Q. P. Liu, and L. Xue, "Supersymmetric SawadaKotera equation: Bäcklund-Darboux transformations and applications," Journal of Nonlinear Mathematical Physics, vol. 25, no. 3, pp. 375-386, 2018.

[9] M. G. Sakar, F. Uludag, and F. Erdogan, "Numerical solution of time-fractional nonlinear PDEs with proportional delays by homotopy perturbation method," Applied Mathematical Modelling, vol. 40, no. 13-14, pp. 6639-6649, 2016.

[10] M. Eslami, B. Fathi Vajargah, M. Mirzazadeh, and A. Biswas, "Application of first integral method to fractional partial differential equations," Indian Journal of Physics, vol. 88, no. 2, pp. 177-184, 2014.

[11] E. M. E. Zayed and Y. A. Amer, "The first integral method and its application for deriving the exact solutions of a higherorder dispersive cubic-quintic nonlinear Schrödinger equation," Computational Mathematics and Modeling, vol. 27, no. 1, pp. 80-94, 2016.

[12] B. Lu, "The first integral method for some time fractional differential equations," Journal of Mathematical Analysis and Applications, vol. 395, no. 2, pp. 684-693, 2012.

[13] J. He, "A new approach to nonlinear partial differential equations," Communications in Nonlinear Science and Numerical Simulation, vol. 2, no. 4, pp. 230-235, 1997.

[14] A. M. Wazwaz and S. A. El-Tantawy, "Optical Gaussons for nonlinear logarithmic Schrödinger equations via the variational iteration method," Optik, vol. 180, pp. 414-418, 2019.

[15] X. F. Yang, Z. C. Deng, and Y. Wei, “A Riccati-Bernoulli subODE method for nonlinear partial differential equations and its application," Advances in Difference Equations, vol. 2015, no. $1,2015$.
[16] X. Si-Liu, L. Jian-Chu, and Y. Lin, "Exact soliton solutions to a generalized nonlinear Schrödinger equation," Communications in Theoretical Physics, vol. 53, no. 1, pp. 159$165,2010$.

[17] X. Li and M. Wang, "A sub-ODE method for finding exact solutions of a generalized $\mathrm{KdV}-\mathrm{mKdV}$ equation with highorder nonlinear terms," Physics Letters A, vol. 361, no. 1-2, pp. 115-118, 2007.

[18] Z. Yan, "Abundant families of Jacobi elliptic function solutions of the (2+1)-dimensional integrable Davey-Stewartson-type equation via a new method," Chaos, Solitons \& Fractals, vol. 18, no. 2, pp. 299-309, 2003.

[19] A. M. Wazwaz, "The tanh method: exact solutions of the sine-Gordon and the sinh-Gordon equations," Applied Mathematics and Computation, vol. 167, no. 2, pp. 1196-1210, 2005.

[20] M. Wang, X. Li, and J. Zhang, "The $\left(G^{\prime} / G\right)$-expansion method and travelling wave solutions of nonlinear evolution equations in mathematical physics," Physics Letters A, vol. 372, no. 4, pp. 417-423, 2008.

[21] A. A. Al-Shawba, K. A. Gepreel, F. A. Abdullah, and A. Azmi, "Abundant closed form solutions of the conformable time fractional Sawada- Kotera-Ito equation using $\left(G^{\prime} /\right.$ G)-expansion method," Results in Physics, vol. 9, pp. 337343, 2018.

[22] R. Hirota, "Exact solution of the Korteweg-de Vries equation for multiple collisions of solitons," Physical Review Letters, vol. 27, no. 18, pp. 1192-1194, 1971.

[23] A. M. Wazwaz and S. A. El-Tantawy, "Solving the $(3+1)$ dimensional KP-Boussinesq and BKP-Boussinesq equations by the simplified Hirota's method," Nonlinear Dynamics, vol. 88, no. 4, pp. 3017-3021, 2017.

[24] M. Gürses and A. Pekcan, "Nonlocal modified KdV equations and their soliton solutions by Hirota Method," Communications in Nonlinear Science and Numerical Simulation, vol. 67, pp. 427-448, 2019.

[25] M. Wang, Y. Zhou, and Z. Li, “Application of a homogeneous balance method to exact solutions of nonlinear equations in mathematical physics," Physics Letters A, vol. 216, no. 1-5, pp. 67-75, 1996.

[26] A. M. Abourabia and Y. A. Eldreeny, "Analytical solution of the complex polymer equation systems via the homogeneous balance method," Physical Science International Journal, vol. 23, no. 4, pp. 1-8, 2019.

[27] M. Eslami, B. Fathi vajargah, and M. Mirzazadeh, "Exact solutions of modified Zakharov-Kuznetsov equation by the homogeneous balance method," Ain Shams Engineering Journal, vol. 5, no. 1, pp. 221-225, 2014.

[28] M. G. Hafez, M. N. Alam, and M. A. Akbar, "Exact traveling wave solutions to the Klein-Gordon equation using the novel $\left(G^{\prime} / G\right)$-expansion method," Results in Physics, vol. 4, pp. 177-184, 2014.

[29] J. Manafian and M. Lakestani, "Solitary wave and periodic wave solutions for Burgers, Fisher, Huxley and combined forms of these equations by the $\left(G^{\prime} / G\right)$-expansion method," Pramana, vol. 85, no. 1, pp. 31-52, 2015.

[30] M. Wang, J. Zhang, and X. Li, “Application of the $\left(G^{\prime} / G\right)$ -expansion to travelling wave solutions of the Broer-Kaup and the approximate long water wave equations," Applied Mathematics and Computation, vol. 206, no. 1, pp. 321-326, 2008. 
[31] E. G. Fan, "Connections among homogeneous balance method, Weiss-Tabor-Carnevale method and Clarkson-Kruskal method," Acta Physica Sinica, vol. 49, pp. 1409-1412, 2000.

[32] F. En-Gui, "Bäcklund transformation and similarity reductions of nonlinear partial differential equations using extended homogeneous balance method," Communications in Theoretical Physics, vol. 35, no. 5, pp. 523-526, 2001. 